\title{
Two Wheel Drive Motorcycle
}

\author{
Ben Jose ${ }^{1}$, Aswin B Vasanth ${ }^{2}$, Adityan $\mathrm{R}^{3}$, Basil George Thomas ${ }^{4}$ \\ 1,2,3,4 (Mechanical Engineering, Saintgits College of Engineering, Kerala, India)
}

\begin{abstract}
Nowadays people concentrate more and more on the comfort and operability of automobiles. Four wheel-drives in cars have become popular and are in demand in the automotive sector. But the advancement in two wheelers was limited. The concept of two wheel drive motorcycles was not successfully and economically implemented. Two wheel drive motorcycle is desirable in situations where there is rough terrain and high inclination roads which are tiresome and difficult to drive with the conventional rear wheel driven motorcycles. The two wheel drive bikes are apt for farmers, military applications, desert drive etc. The proposed design provide economical and user friendly two wheel drive. The use of chain drive and sprockets help in reduction of power loss during transmission of power from the engine to front and rear wheels. The lightweight 'allmechanical system' is used to obtain two wheel-drive. Under optimum traction conditions, the rear wheel is actually driving faster than the front wheel and the one-way clutch within the system allow the front wheel to freewheel under these conditions. At this point, the two wheel drive system is effectively passive. Though the front drive system is turning, it is not actually transferring power to the front wheel. When the rear wheel loses traction, the drive ratio, relative to your forward speed, changes. The two wheel drive system engages transferring power to the front wheel until traction is re-established at the rear wheel.
\end{abstract}

Keywords: Two wheel drive, front wheel drive, bikes for farmland, military bikes, adventure bike

\section{INTRODUCTION}

The automobile companies are bringing out many two wheeler models every year around the world. Nowadays importance is given more to function and performance than the beauty of the vehicle. Motorcycles for instant are preferred for transport as well as adventure riding and other applications. The conventional rear wheel drive motorcycle uses chains to transmit power from the engine to the rear wheel. But their applications are limited. The load carrying capacity is limited. The climbing capacity is also limited as there is torque only at the rear wheel. If the rear wheel slips, the vehicle does not move. This concludes that the conventional motorcycles cannot be used at ease in climbing high inclination roads, driving along muddy roads, desert roads, farm lands, mountains etc. These motorcycles are also not suited in off roads and adverse terrains.

Two wheel drive system is a solution to these limitations. In this system power is given to both the front and the rear wheel. That is, if the rear wheel slips the power given to the front wheel is enough to move the motorcycle forward. This system is similar to the four wheel drive system used in cars. With the two wheel drive system the load carrying capacity, traction and cornering ability of two wheelers are increased. It also results in unmatched hill climbing ability when compared with conventional motorcycles available in the market today. Because of this they are preferred for farm lands and military applications (reconnaissance missions). It is evident from the fact that a decade ago the two wheel drive motorcycles were limited to dirt races and mountain races. But now they are being wanted for farm and military applications and more companies are showing interest in the two wheel drive system on motorcycles.

Two wheel drive motorcycles that are already in the market are not accepted well by the customers due to factors like high cost and large size owing to high capacity engine and the complex mechanisms involved for transmission, high fuel consumption etc.

The aim of our project is to fabricate a two wheel drive motorcycle at low cost. The motorcycle would be using chains to transmit power from the engine to both the wheels so as to make the system simpler. A constant velocity joint is used at front wheel to transmit the power between shafts rotating in different axes. A freewheel is used to make the engagement of the front wheel drive automatic. Power is given to the front wheel only when the rear wheel slips.

\section{PREVIOUS DESIGNS}

Many companies have designed two wheel drive bikes. Some of them are already in production and are available in the market. Of which the companies Rokon, Yamaha, Christini are of more importance.

Rokon: Rokon is a company with several models of two wheel drive bikes in the market. The models are bulky in size and heavy. The bikes have big tires and a big frame to support it. The company set out to build the ultimate off road vehicle in the 1950s. It had to go anywhere and be a rugged workhorse. With this in mind a two-wheel drive vehicle with hollow wheels was designed and produced. Some call it a bike but the name Moto 
tractor more closely identifies it. This is because of its huge size. Power to both wheels in a lightweight yet strong frame design gave riders nimble and versatile traction off road.

Christini: Christini Motors is one of the leading manufacturers of two wheel drive bikes in the world. Chistini's patented mechanical all wheel drive system delivers power from the motorcycle transmission to the front wheel through a series of chains and shafts. There is no energy robbing hydraulics involved. The lightweight all-mechanical system works similar to that of AWD systems found on four wheeled vehicles.

The AWD system (powering the front wheel) is driven at a slightly lower rate than the rear wheel (approximately 80\%). Under optimum traction conditions, the rear wheel is actually driving faster than the front AWD system. One-way clutches within the front hub allow the front wheel to freewheel under these conditions. At this point, the AWD system is effectively passive. Though the front AWD system is turning, it is not actually transferring power to the front wheel. When the rear wheel loses traction, the drive ratio, relative to your forward speed, changes. The AWD system engages, transferring power to the front wheel until traction is reestablished at the rear wheel. There are different models in the market manufactured by Christini. It also has a military AWD bike designed for the American Military.

Two wheel drive bike by HCE students: It was designed by a team of students from Madras-based Hindustan College of Engineering (HCE), \{Rachit Aggarwal from Delhi, Arpit Tandon from Muradabad, UP, Pawan J B Rana from Nepal and Lakshman Sreedhar from Chennai \}. It was designed and developed to transmit power to the front wheel using a combination of shaft and chain drive from the engine output. They used a Kawasaki Bajaj 100 RTZ and incorporated a selector mechanism obtained from a Jeep's transfer case. Thus the engagement of two wheel drive was manually done. They used a propeller shaft with two integrated CV joints, three bevel gears, two sprockets, chains etc.

\section{DRAWBACKS OF AVAILABLE DESIGNS}

The current available designs have several disadvantages. The cheapest one among them costs above $\$ 8000$ and common people cannot afford it. Also the transmission systems employed in such designs are more complex. The current designs available are using hydraulic transmissions, which thus have high power loss. The very big size of these bikes makes them suitable only for limited uses like race events etc.

One of the available designs is ROKON 2WD bike, which have got a full time two wheel drive system employed. The full time two wheel drive mechanism has considerably less fuel economy. And also the size of these bikes is very high which limits the use. Another design available is YAMAHA YZ250, which is a production bike by the famous two wheel manufacturers, YAMAHA. These bikes are used only for mountain races and are equipped with high capacity engines. Also the cost of the above two designs are very high. CHRISTINI BIKES, one of the leading companies in manufacturing two wheel drive bike, they are manufacturing $2 \mathrm{WD}$ bikes with medium capacity engines. Even they are manufacturing medium capacity bikes, the cost is still very high. The transmission used by CHRISTINI BIKES is a combination of chain drives and shaft drives and therefore the transmission losses are less compared to other designs

\section{DESIGN}

The front wheel should be given a higher sprocket ratio than the rear that is the front wheel rotates at a lower speed than the rear. This condition is given to have a differential effect when the bike is taking a turn. When a bike takes a turn the bike turns with respect to a point on the ground. Thus the front wheel will be turned at an angle to the bike and the rear wheel will be in line with the bike. If the front wheels drives faster than the rear, the vehicle will experience a pulling effect. This will make uncomfortable for the rider to make correct balance at turns. A faster rear wheel will also give directional stability while cruising at high speeds.

Since the front wheel is at a lower speed, to avoid slip a free wheel should be used. A free wheel is a one-way clutch which transfers power only in one direction. Thus under optimum traction conditions, the rear wheel is actually driving faster than the front AWD system. One-way clutches within the front hub allow the front wheel to freewheel under these conditions. At this point, the AWD system is effectively passive and no power will be transmitted to the front. Thus the bike will act as a RWD vehicle. Thus the fuel consumption will not increase.

The way the automatic engagement works is like pedaling a bicycle downhill. You are pedaling, but because of gravity (acting like the rear drive) the bike is travelling faster than you are delivering power. When you get to the bottom of the hill and slow down (similar to what happens when the rear wheel spins), you will begin to power the bike again. Thus only when the rear wheel loses traction on the road (the rear wheel spinning simply without moving the vehicle) the front wheel should engage and should drive the vehicle. Thus the AWD system engages only when required.

A small size $\mathrm{CV}$ joint is required which can be accommodated within the space between the fork. Also it should be cheap and should give the required angle of turn corresponding to the handle. The front wheel 
should have a sprocket to receive the power from the engine. A chain having the corresponding pitch and thickness is required.

\subsection{Vehicle selected}

The selected vehicle is a TVS 50 motorcycle. It has a 50cc two stroke engine, a centrifugal clutch, the clutch output is connected to a pulley through belt transmission and a sprocket is connected to a sprocket which drives the rear wheel through a chain drive in the ratio $4: 1$. It has a simple tubular frame. Thus it is easy to incorporate the two wheel drive system in this motorcycle. Moreover the cost is less. Thus for reducing the cost, for the ease of fabrication and simple design we have chosen India's first two seater moped: TVS 50

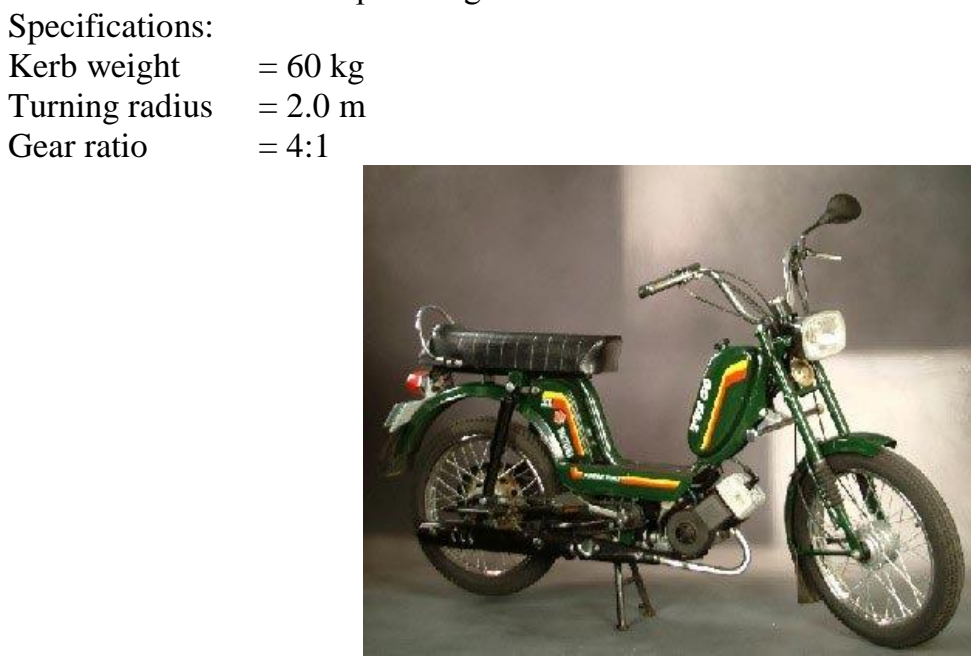

Fig 4 (a) TVS 50 motorcycle

\begin{tabular}{|l|l|}
\hline Type & 2 stroke single cylinder \\
\hline Bore $\mathrm{x}$ Stroke $(\mathrm{mm})$ & $46 \times 42$ \\
\hline Displacement $(\mathrm{cc})$ & 49.9 \\
\hline Max. Power & $2.61 \mathrm{KW}(3.5 \mathrm{bhp}) @ 5000 \mathrm{rpm}$ \\
\hline Torque (Nm) & $5.0 @ 3750 \mathrm{rpm}$ \\
\hline & \\
\hline Tire size ( front) & $5.4 \quad \mathrm{X} 16$ \\
\hline Tire size ( rear) & $2.5 \times 16$ \\
\hline
\end{tabular}

\subsection{Design process}

To take power to the front:

- A chain drive is taken from the pulley to a position below the handle.

- The end of this chain drive is coupled to one side of a tripod CV joint.

- A sprocket is fitted at the right end of the $\mathrm{CV}$ joint.

- A chain drive is taken from this sprocket to the sprocket at the front wheel.

- The length of the pulley axis is increased.

- An extra sprocket (number of teeth: 16) is added to the end of the pulley.

- The CV joint is positioned below the handle so that the two shafts of the CV joint meet exactly on the axis of steering.

- A free wheel (number of teeth: 18) is attached to the left shaft of the CV joint. A free wheel is a one way clutch which transmits power in only one direction, and in opposite direction there will be no power transmission. It is commonly used as the sprocket at the rear wheel of bicycles.

- At the other end of the CV joint another sprocket (number of teeth: 13), with greater pitch, is fixed. The front wheel of the motorcycle is replaced by the rear wheel of the similar motorcycle having the rear sprocket (number of teeth: 52).

- A chain drive is taken from the right side sprocket of the CV joint to the sprocket at the front wheel. 


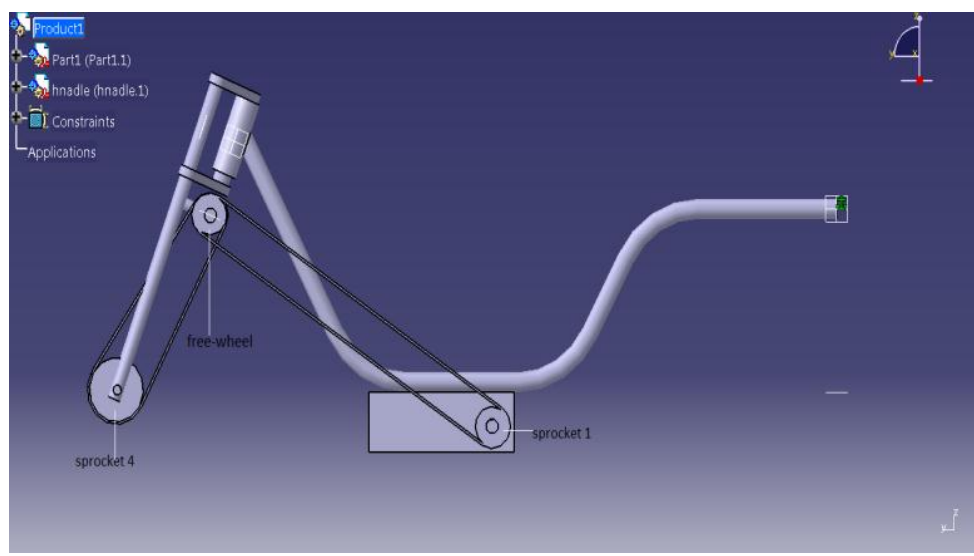

Fig. 4(b) Side view of the design

The final sprocket ratio from the sprocket at the engine side to the front wheel is $4.5: 1$. But the ratio to the rear wheels is $4: 1$. Thus the rear wheel is driven at a speed slightly greater than the front. The velocity ratio from the rear to the front wheels is 1.125:1.

A CV joint is used instead of a simple universal joint because the universal joint when turned above an angle greater than $18^{\circ} \mathrm{s}$ the output shaft will not give a constant velocity, which is not desirable in our system. But for a $\mathrm{CV}$ joint the angular velocity is always constant about $55^{\circ} \mathrm{s}$.

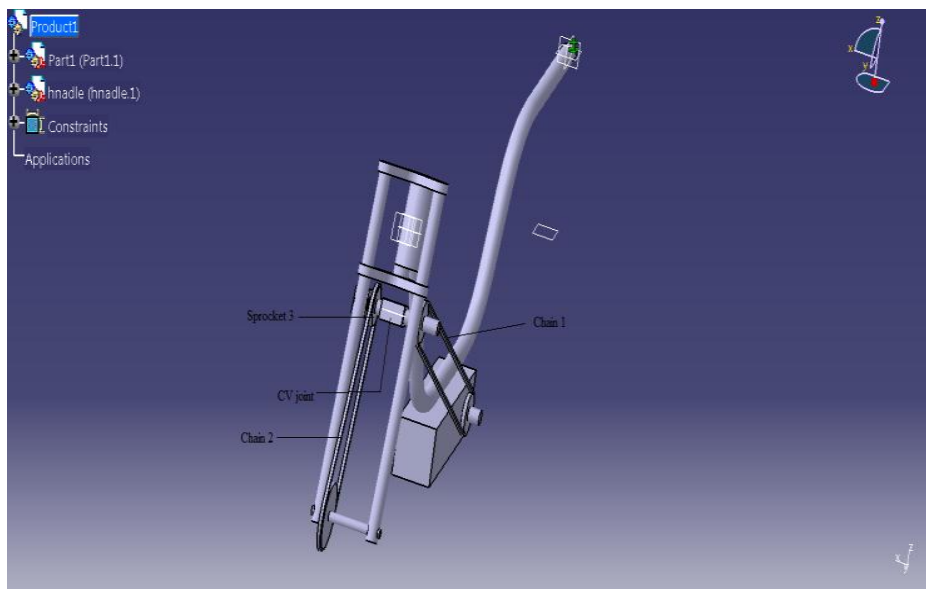

Fig 4(c) Isometric view of the design

\subsection{Design for gear ratio}

Design has to be such that power is to be transmitted to front side only when the back wheel slips. To obtain this we use freewheel and a higher gear ratio at front side than the rear side gear ratio. The calculation for gear ratio is done with following considerations:

1. Freewheel used is that of bicycle having pitch $12.7 \mathrm{~mm}$ and number of teeth 18 .

Freewheel was considered as the driven sprocket from the engine side.

2. Front wheel was replaced with back wheel of TVS 50 motorcycle. It contained hub with sprocket mounted on it. Pitch of the sprocket is $13.7 \mathrm{~mm}$ and contained 52 teeth.

Design of gear ratio is done in three stages:

Stage 1: From engine side to the freewheel

As freewheel (driven sprocket) used was that of the bicycle, commonly available in the market, pitch and number of teeth was fixed.

Pitch $\quad=12.7 \mathrm{~mm}$

Number of teeth on driven sprocket $\quad=18$

To obtain higher gear ratio choose driving sprocket such that

Pitch $\quad=12.7 \mathrm{~mm}$

Number of teeth on driver sprocket $\quad=16$

Gear ratio $i_{1}$

$=18 / 16=1.125: 1$ 
Stage 2: Power transmission from CV joint shaft to the front wheel

As the front wheel contained sprocket (driven sprocket) of pitch $13.4 \mathrm{~mm}$ and 52 teeth we choose driver sprocket such that

Pitch

Number of teeth on driver sprocket

$$
\begin{array}{ll} 
& =13.4 \mathrm{~mm} \\
& =13 \\
& =52 / 13 \\
& =4: 1 \\
& =\mathrm{i}_{1} * \mathrm{i}_{2} \\
& =1.125 * 4 \\
& =4.5: 1
\end{array}
$$

\subsection{Materials Required}

\section{Constant velocity (CV) joint}

Constant velocity joint has to be incorporated within the forks of the motorcycle. So CV joint of Tata Nano car is chosen as it was the smallest one available in the market. It is a tripod type CV joint .These joints are used at the inboard end of car drive shafts. This joint has a three-pointer yoke attached to the shaft, which has barrel-shaped roller bearings on the ends. These fit into a cup with three matching grooves on the casing and allow an axial 'plunge' movement of the shaft, so that engine rocking and other effects do not preload the bearings.

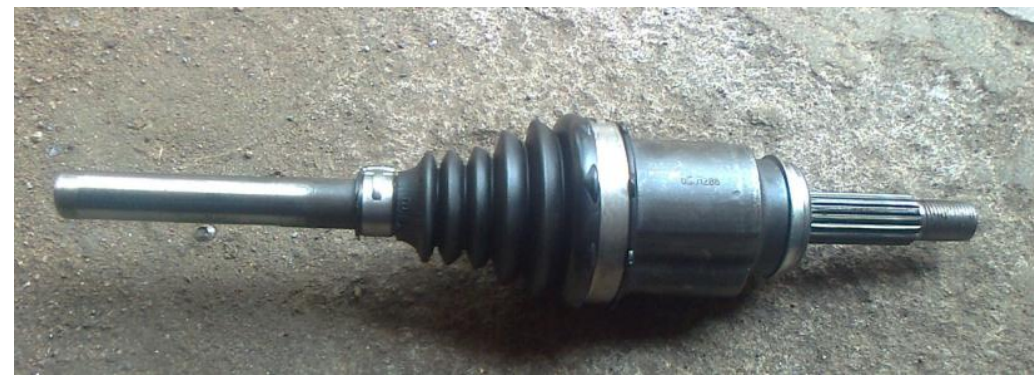

Fig 4(d) CV joint used in Tata Nano

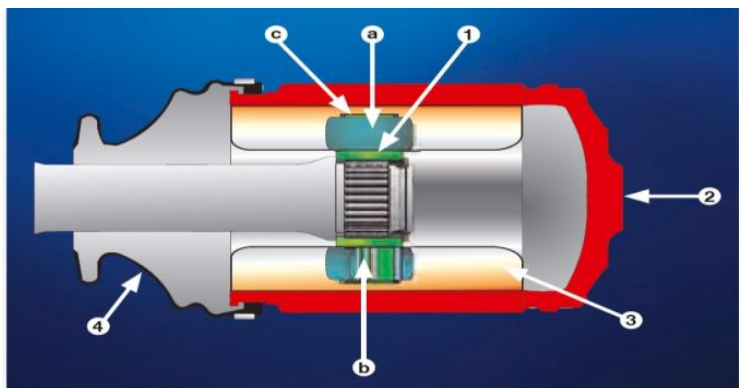

\section{Free wheel}

Fig 4(e) crossectional view of the above $\mathrm{CV}$ joint

A free wheel is used in order to make the two wheel engagement automatic. The free wheel used is the commonly available one for bicycles.

$\begin{array}{ll}\text { Specifications: } & =12.7 \mathrm{~mm} \\ \text { Pitch } & =18 \\ \text { Number of teeth } & =175 \mathrm{~g} . \\ \text { Weight } & : \text { Steel. }\end{array}$




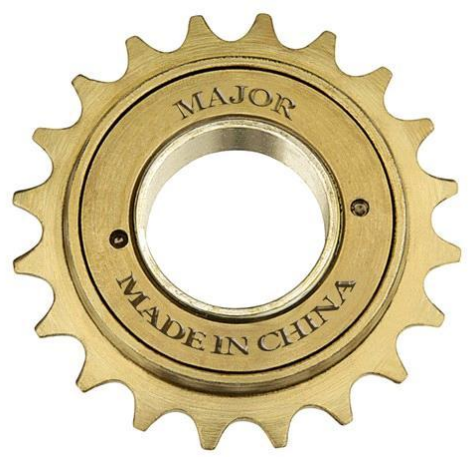

Fig4(f) free wheel

\section{Sprockets}

Sprockets and chains are used to transmit power from engine output to the front wheel. In the design three sprockets were used

a. $\quad$ Sprocket 1:

The sprocket 1 is coupled to the engine output shaft and delivers power to the freewheel.

Specifications:

$\begin{array}{ll}\text { Pitch } & =12.7 \mathrm{~mm} \\ \text { Number of teeth } & =16 .\end{array}$

Material : Steel C45

b. $\quad$ Sprocket 2:

Sprocket 2 obtains power from CV joint and transmits it to the front wheel.

Specifications:

$\begin{array}{ll}\text { Pitch } & =13.4 \mathrm{~mm} \\ \text { Number of teeth } & =13\end{array}$

Material : Steel C45

c. $\quad$ Sprocket 3 :

This sprocket is mounted on the front wheel and obtains power from the sprocket 2 . This sprocket drives the front wheel when two wheel drive is engaged.

Specifications:

Pitch

$=13.4 \mathrm{~mm}$

Number of teeth $\quad=52$

Material

: Steel C45

4. Chain

Chains play an important role as all the power transmission is using sprocket and chains. Two chains of different pitches are used.

a. Chain 1 , Pitch $=12.7 \mathrm{~mm}$

Design for number of links:

Pitch, p

Centre distance between sprockets $X$

Number of teeth on driven sprocket $T_{1}$
Number of teeth on driver sprocket $T_{2}$

Number of links K

$$
\begin{aligned}
& =12.7 \mathrm{~mm} \\
\mathrm{X} & =545 \mathrm{~mm} \\
\mathrm{~T}_{1} & =16 \\
\mathrm{~T}_{2} & =18 \\
& =\left(\mathrm{T}_{1}+\mathrm{T}_{2}\right) / 2+(2 \mathrm{X} / \mathrm{p})+(\mathrm{p} / \mathrm{X}) *\left[\left(\mathrm{~T}_{2}-\mathrm{T}_{1}\right) /(2 \pi)\right]^{2} \\
& =(16+18) / 2+[(2 * 545) / 12.7]+(12.7 / 545) *[(18- \\
& \left.\quad 16)^{2} / 4 \pi^{2}\right] \\
\mathrm{K} & =17+85.83+2.36 \times 10-3 \\
& =102 \text { links }
\end{aligned}
$$


b. Chain 2, Pitch $=13.4 \mathrm{~mm}$

Design for number of links:

Pitch, $p$

Centre distance between sprockets $\mathrm{X}$

$=13.4 \mathrm{~mm}$

Number of teeth on driven sprocket $T_{1}$

$=340 \mathrm{~mm}$

Number of teeth on driver sprocket $\mathrm{T}_{2}$

$=52$

Number of links K

$$
\begin{aligned}
& =13 \\
& =\left(\mathrm{T}_{1}+\mathrm{T}_{2}\right) / 2+(2 \mathrm{X} / \mathrm{p})+(\mathrm{p} / \mathrm{X}) *\left[\left(\mathrm{~T}_{2}-\mathrm{T}_{1}\right) /(2 \pi)\right]^{2}
\end{aligned}
$$$$
=(52+13) / 2+[(2 * 340) / 13.4]+(13.4 / 340) *[(52-
$$$$
\left.13)^{2} / 4 \pi^{2}\right]
$$$$
=32.5+50.75+1.52
$$

$\mathrm{K} \quad=84.76$

$\mathrm{K}=86$ links

\section{Mild steel}

Mild steel is required for fabricating the support assembly for CV joint axle and sprockets on the shaft. $\mathrm{CV}$ joint axle and sprocket assembly are mounted below the handle such that the turning axis of the $\mathrm{CV}$ joint coincides with the turning axis of the vehicle. Also mild steel is required for providing bearing seatings for three bearings.

\section{Bearings}

Bearings are used to support the $\mathrm{CV}$ joint axle at accurate positions. Three ball bearings are used having bearing number SKF 6203.

\section{Problems Encountered}

During the progress of the project many problems were encountered. Some of them are:

- The vehicle was not turning the required angle due to offset of the CV joint turning axis and vehicle axis.

- Heavy Vibration.

- Chain drive failure due to chain slackening at rough terrain travel.

- Failure of chain drive at higher speeds.

- Increased turning radius of the motorcycle because of restriction provided by the CV joint rubber bushing. .

\section{Solutions}

- To reduce the offset in the CV joint axis and steering axis, CV joint was shortened in length (excess length removed) and found successful.

- To reduce vibration additional bearing was introduced at the input shaft of CV joint and found successful.

- To obtain rigidity extra support was welded to the support assembly.

- Misalignment of the chain was rectified.

- Bushing was removed and low resistance bushing was incorporated.

\section{CONCLUSION}

The rear wheel drive motorcycle which we initially started with was successfully converted into a two wheel drive motorcycle with automatic engagement within the given span of time. Automatic engagement of the front wheel was made possible by using a free wheel. At optimum running condition the vehicle behaves same as a rear wheel drive vehicle and do not produce any problems. The turning radius of the vehicle is found to be 2.5 meter, with an increase of only 0.5 meter from the initial condition. Since we made simple design the weight of the vehicle increased by only $6 \mathrm{~kg}$ and hence there is no much variation in the fuel efficiency of the motorcycle.

As we used chain drive, the power loss is very less when compared to the older designs by Yamaha, Rokon etc. The motorcycle effectively negotiates steep inclination which it couldn't make up in the normal rear wheel drive and moreover, it navigates with minimum effort in muddy and slippery condition. The load carrying capacity of the motorcycle has also increased slightly.

We obtained a higher sprocket ratio at the front wheel than that of the back wheel and hence the front wheel rotates at a lower rpm than the rear wheel. During normal run by the action of the free wheel, no power is utilized by the front wheel and only during the slip of rear wheel the front wheel drive engage its action. So we could effectively create an automatic engagement of the front wheel drive.

There can be a lot of improvisation in our design and can launch the two wheel drive bikes in the market. The turning radius of the two wheel drive motorcycle is found to be $2.5 \mathrm{~m}$, but in the original vehicle is $2 \mathrm{~m}$. The increase in the turning radius is because of the limitation of the $\mathrm{CV}$ joint and a slight offset between the 
steering axis and the $\mathrm{CV}$ joint turning axis. By using a smaller $\mathrm{CV}$ joint designed for two wheel drive motor cycles the turning radius can be reduced. Also the design proposed under the title Future scope can be implemented.

The increase in vibration as the speed of the motorcycle increases is due to low rigidity of support assembly, using a more rigid and robust support frame for the CV joint-freewheel assembly the vibrations can be reduced and vehicle can attain higher speeds.

\section{Acknowledgements}

First of all we express our sincere thanks and indebtedness to GOD almighty for his grace and bounteous blessings for completing this project in a benefiting manner.

We are highly grateful to Mr. M. C. Philipose, Principal, Saintgits College of Engineering, Pathamuttom, Kottayam Kerala, India for allowing us to use the college facilities for completing our project work.

We would like to express our wholehearted gratitude to our guide Mr. Sajan Thomas, Professor, Mechanical Engineering Department for his valuable guidance and inspiration throughout the course of the project. We also take this opportunity to thank all the staff members in the Mechanical Laboratory, Saintgits college of Engineering.

This acknowledgement would be incomplete without expressing our gratitude to our parents who act as a beacon light and inspired us unconditionally and to our friends who motivated us during the tenure of study.

\section{Books:}

\section{REFERENCES}

[1] William H. Crouse and Donald L Anglin, Automotive Mechanics(New York: McGraw-Hill, 1994).

[2] Joseph Edward Shigley, Mechanical Engineering Design(New Delhi: Tata McGrag-Hill Education, 2001).

[3] R.S. Khurmi and J.K. Gupta, A Textbook of Machine Design(New Delhi: Eurasia Publishing House, 1979)

\section{Chapters in Books:}

[4] K Mahadevan and K Balaveera Reddy, Design Data Handbook, $3^{\text {rd }}$ edition(New Delhi: CBS Publishers, 1987) Chapters $1,2,3,5,15,16$ and 20 